\title{
Some Properties for an Integral Operator Defined by Generalized Hypergeometric Function
}

\author{
Ibtisam Aldawish and Maslina Darus
}

School of Mathematical Sciences, Faculty of Science and Technology, Universiti Kebangsaan Malaysia, 43600 Bangi, Selangor, Malaysia

Correspondence should be addressed to Maslina Darus; maslina@ukm.edu.my

Received 10 May 2014; Accepted 4 July 2014; Published 4 August 2014

Academic Editor: Song Cen

Copyright ( 2014 I. Aldawish and M. Darus. This is an open access article distributed under the Creative Commons Attribution License, which permits unrestricted use, distribution, and reproduction in any medium, provided the original work is properly cited.

We define a new general integral operator for meromorphic functions involving the generalized hypergeometric function. Furthermore, we study the characterization and other properties for this operator.

\section{Introduction}

Let $\Omega$ denote the class of functions of the form

$$
f(z)=\frac{1}{z}+\sum_{k=1}^{\infty} a_{k} z^{k}
$$

which are analytic in the punctured open unit disk $\mathbb{U}^{*}=\{z$ : $z \in \mathbb{C}, 0<|z|<1\}=\mathbb{U} \backslash\{0\}$.

We say that a function $f \in \Omega$ is meromorphic starlike of order $\eta(0 \leq \eta<1)$ and belongs to the class $\Omega^{*}(\eta)$, if it satisfies the following inequality:

$$
-\mathfrak{R e}\left(\frac{z f^{\prime}(z)}{f(z)}\right)>\eta
$$

A function $f \in \Omega$ is a meromorphic convex function of order $\eta(0 \leq \eta<1)$, if $f$ satisfies the following inequality:

$$
-\mathfrak{R e}\left(1+\frac{z f^{\prime \prime}(z)}{f^{\prime}(z)}\right)>\eta,
$$

and we denote this class by $\Omega_{k}(\eta)$.

Also, let $\Omega_{N}(\xi)(\xi>1, z \in \mathbb{U})$ be the subclass of $\Omega$, consisting of the functions $f$, which satisfy the inequality

$$
-\mathfrak{R e}\left(1+\frac{z f^{\prime \prime}(z)}{f^{\prime}(z)}\right)<\xi \quad(\xi>1, z \in \mathbb{U}),
$$

where the subclass $\Omega_{N}(\xi)$ was introduced and studied by Wang et al. [1] and Nehari and Netanyahu [2].

A $q$-hypergeometric function is a power series in one complex variable $z$ with power series coefficients which depend (apart from q) on $r$ complex upper parameters $\alpha_{i}$, $\beta_{j},\left(i=1, \ldots, r, j=1, \ldots, s, \alpha_{i} \in \mathbb{C}, \beta_{j} \in \mathbb{C} \backslash\{0,-1,-2, \ldots\}\right)$ as follows:

$$
\begin{aligned}
& \mathbb{V}\left(\alpha_{1}, \ldots, \alpha_{r} ; \beta_{1}, \ldots, \beta_{s}, q, z\right) \\
& \quad=\sum_{k=0}^{\infty} \frac{\left(\alpha_{1}, q\right)_{k} \cdots\left(\alpha_{r}, q\right)_{k}}{(q, q)_{k}\left(\beta_{1}, q\right)_{k} \cdots\left(\beta_{s}, q\right)_{k}}\left[(-1)^{k} q^{\left.\left(\begin{array}{l}
k \\
2
\end{array}\right)\right]^{1+s-r} z^{k},}\right.
\end{aligned}
$$

with $\left(\begin{array}{l}k \\ 2\end{array}\right)=k(k-1) / 2$, where $q \neq 0$ when $r>s+1,(r, s \in$ $\left.\mathbb{N}_{0}=\mathbb{N} \cup\{0\}\right)$, and $(\alpha, q)_{k}$ is the Pochhammer symbol $(\alpha)_{k}$ defined by

$$
\begin{aligned}
& (\alpha, q)_{k} \\
& \quad= \begin{cases}1, & k=0 ; \\
(1-\alpha)(1-\alpha q)\left(1-\alpha q^{2}\right) \cdots\left(1-\alpha q^{k-1}\right), & k \in \mathbb{N} .\end{cases}
\end{aligned}
$$

Tracing back the history of basic hypergeometric series defined by (5) has brought us to Heine dated in 1846. Therefore it is sometimes called Heine's series. For brief survey on $q$-hypergeometric functions, one may refer to [3-5] (see also [6-8]). 
For $z \in \mathbb{U},|q|<1$, and $r=s+1$, the basic hypergeometric function defined in (5) takes the form

$$
\begin{aligned}
{ }_{r} \Lambda_{s} & \left(\alpha_{1}, \ldots, \alpha_{r} ; \beta_{1}, \ldots, \beta_{s}, q, z\right) \\
& =\sum_{k=0}^{\infty} \frac{\left(\alpha_{1} ; q\right)_{k} \cdots\left(\alpha_{r} ; q\right)_{k}}{(q ; q)_{k}\left(\beta_{1} ; q\right)_{k} \cdots\left(\beta_{s} ; q\right)_{k}} z^{k}
\end{aligned}
$$

which converges absolutely in the open unit disk $\mathbb{U}$.

Corresponding to the function ${ }_{r} \Lambda_{s}\left(\alpha_{1}, \ldots, \alpha_{r} ; \beta_{1}, \ldots, \beta_{s}\right.$, $q, z)$, let

$$
\begin{gathered}
r \Psi_{s}\left(\alpha_{1}, \ldots, \alpha_{r} ; \beta_{1}, \ldots, \beta_{s}, q, z\right)=\frac{1}{z} \\
{ }_{r} \Lambda_{s}\left(\alpha_{1}, \ldots, \alpha_{r} ; \beta_{1}, \ldots, \beta_{s}, q, z\right) \\
=\frac{1}{z}+\sum_{k=1}^{\infty} \frac{\left(\alpha_{1}, q\right)_{k+1} \cdots\left(\alpha_{r}, q\right)_{k+1}}{(q, q)_{k+1}\left(\beta_{1}, q\right)_{k+1} \cdots\left(\beta_{s}, q\right)_{k+1}} z^{k}
\end{gathered}
$$

Analogous to the differential operator defined in [9] which involves the $q$-hypergeometric functions on the normalized analytic functions, we define the following differential operator $\mathcal{N}_{r, s}^{n}\left[\alpha_{i}, \beta_{j} ; q\right]: \Omega \rightarrow \Omega$ on the space of meromorphic functions in the class $\Omega$ by

$$
\begin{aligned}
\mathcal{N}_{r, s}^{0}\left[a_{i}, b_{j} ; q\right] f(z)= & f(z) *{ }_{r} \Psi_{s}\left(a_{i}, b_{j} ; q ; z\right) \\
\mathcal{N}_{r, s}^{1}\left[a_{i}, b_{j} ; q\right] f(z)= & (1-\lambda) f(z) *{ }_{r} \Psi_{s}\left(a_{i}, b_{j} ; q ; z\right) \\
& +\lambda z D_{q}\left(f(z) *{ }_{r} \Psi_{s}\left(a_{i}, b_{j} ; q ; z\right)\right) \\
\vdots & \\
\mathcal{N}_{r, s}^{n}\left[a_{i}, b_{j} ; q\right] f(z)= & \mathcal{N}_{r, s}^{1}\left(\mathcal{N}_{r, s}^{n-1}(f(z))\right) .
\end{aligned}
$$

If $f \in \Omega$, then from (9) we may deduce that

$$
\mathcal{N}_{r, s}^{n}\left[a_{i}, b_{j} ; q\right] f(z)=\frac{1}{z}+\sum_{k=1}^{\infty}[1+(k-1) \lambda]^{n} \Upsilon_{k} a_{k} z^{k}
$$

where

$$
\Upsilon_{k}=\frac{\left(\alpha_{1}, q\right)_{k+1} \cdots\left(\alpha_{r}, q\right)_{k+1}}{(q, q)_{k+1}\left(\beta_{1}, q\right)_{k+1} \cdots\left(\beta_{s}, q\right)_{k+1}}
$$

$\lambda \geq 0$, and $n \in \mathbb{N}_{0}=\mathbb{N} \cup\{0\}$.

It should be remarked that the operator given by (10) is a generalization of many other operators considered earlier; for example, consider the following.

(i) For $\lambda=1, r=1, s=0, \alpha_{1}=q, q \rightarrow 1$ the operator $\mathcal{N}_{1,0}[q, q] f(z)=I^{n} f(z)$ was defined by Frasin and Darus [10] and studied by El-Ashwah and Aouf [11].

(ii) For $n=0, \alpha_{i}=q^{\alpha_{i}}, \beta_{j}=q^{\beta_{j}}, \alpha_{i}>0, \beta_{j}>0,(i=$ $1, \ldots, r ; j=1, \ldots, s, r=s+1), q \rightarrow 1$ the operator $\mathcal{N}_{r, s}\left[q^{\alpha_{i}}, q^{\beta_{j}}, q\right] f(z)=\mathscr{H}_{r, s}\left[\alpha_{i}, \beta_{j}\right] f(z)$ was imposed by Liu and Srivastava [12]. (iii) For $n=0, r=2, s=1, \alpha_{2}=q, q \rightarrow 1$, the operator $\mathcal{N}_{2,1}\left[\alpha_{1}, q, \beta_{1}, q\right] f(z)=\mathcal{N}\left[\alpha_{1} ; \beta_{1}\right] f(z)$ was introduced and studied by Liu and Srivastava [13]. Further, we note in passing that this operator $\mathcal{N}\left[\alpha_{1} ; \beta_{1}\right] f(z)$ is closely related to the Carlson-Shaffer operator $\mathcal{N}\left[\alpha_{1} ; \beta_{1}\right] f(z)$ defined on the space of analytic univalent functions in $\mathbb{U}$.

(iv) For $n=0, r=1, s=0, \alpha_{1}=\lambda+1, q \rightarrow 1$, the operator $\mathcal{N}_{1,0}[\lambda+1, q] f(z)=\mathscr{D}^{\lambda} f(z)=\left(1 / z(1-z)^{\lambda+1}\right) *$ $f(z)(\lambda>-1)$, where $\mathscr{D}^{\lambda}$ is the differential operator, was introduced by Ganigi and Uralegaddi [14], and then it was generalized by Yang [15].

Now, making use of the differential operator $\mathcal{N}_{r, s}^{n}\left[\alpha_{i}, \beta_{j} ; q\right]$ given by (10), we introduce the following integral operator in the class $\Omega$.

Definition 1. Let $n \in \mathbb{N}, i \in\{1,2, \ldots, n\}, \delta_{i}>0$. We define the integral operator $l_{\delta_{1}, \ldots, \delta_{n}}(z): \Omega^{n} \rightarrow \Omega$ by

$$
\begin{aligned}
l_{\delta_{1}, \ldots, \delta_{n}}(z) & \\
= & \frac{1}{z^{2}} \int_{0}^{z}\left(-u^{2}\left(\mathcal{N}_{r, s}^{n}\left[\alpha_{i}, \beta_{j} ; q\right] f_{1}\right)^{\prime}(u)\right)^{\delta_{1}} \\
& \ldots\left(-u^{2}\left(\mathcal{N}_{r, s}^{n}\left[\alpha_{i}, \beta_{j} ; q\right] f_{n}\right)^{\prime}(u)\right)^{\delta_{n}} d u .
\end{aligned}
$$

Remark 2. Note that for $n=0, r=1, s=0, \alpha_{1}=q$, we obtain the integral operator defined by Mohammed and Darus [16]; see also [17-19].

With the aid of differential operator $\mathcal{N}_{r, s}^{n}\left[\alpha_{i}, \beta_{j} ; q\right] f(z)$ given by (10), we define a new subclass of functions in $\Omega$ as follows.

Definition 3. Let a function $f \in \Omega$ be analytic in $\mathbb{U}^{*}$. Then $f$ is in the class $\Omega_{k}\left(\alpha_{i}, \beta_{j} ; q, \eta\right)$ if, and only if, $f$ satisfies

$$
-\mathfrak{R e}\left\{1+\frac{z\left(\mathcal{N}_{r, s}^{n}\left[\alpha_{i}, \beta_{j} ; q\right] f\right)^{\prime \prime}(z)}{\left(\mathcal{N}_{r, s}^{n}\left[\alpha_{i}, \beta_{j} ; q\right] f\right)^{\prime}(z)}\right)>\eta,
$$

where $\mathcal{N}_{r, s}^{n}\left[\alpha_{i}, \beta_{j} ; q\right] f$ is defined in (10) and $0 \leq \eta<1$.

Definition 4. Let a function $f \in \Omega$ be analytic in $\mathbb{U}^{*}$. Then $f$ is in the class $\Omega_{r, s} \mathscr{K}\left(\alpha_{i}, \beta_{j}, q, \eta, b\right)$ if, and only if, $f$ satisfies

$$
\mathfrak{R e}\left\{1-\frac{1}{b}\left(\frac{z\left(\mathcal{N}_{r, s}^{n}\left[\alpha_{i}, \beta_{j} ; q\right] f\right)^{\prime \prime}(z)}{\left(\mathcal{N}_{r, s}^{n}\left[\alpha_{i}, \beta_{j} ; q\right] f\right)^{\prime}(z)}+2\right)\right\}>\eta,
$$

where $\mathcal{N}_{r, s}^{n}\left[\alpha_{i}, \beta_{j} ; q\right] f$ is defined in (10) and $b \in \mathbb{C} \backslash\{0\}, 0 \leq$ $\eta<1$. 
Definition 5. Let a function $f \in \Omega$ be analytic in $\mathbb{U}^{*}$. Then $f$ is in the class $\Omega_{r, s} \mathscr{K} \mathscr{U}\left(\alpha_{i}, \beta_{j} ; q, \alpha, \eta, b\right)$ if, and only if, $f$ satisfies

$$
\begin{gathered}
\mathfrak{R e}\left\{1-\frac{1}{b}\left(\frac{z\left(\mathcal{N}_{r, s}^{n}\left[\alpha_{i}, \beta_{j} ; q\right] f\right)^{\prime \prime}(z)}{\left(\mathcal{N}_{r, s}^{n}\left[\alpha_{i}, \beta_{j} ; q\right] f\right)^{\prime}(z)}+2\right)\right\} \\
>\alpha\left|\frac{1}{b}\left(\frac{z\left(\mathcal{N}_{r, s}^{n}\left[\alpha_{i}, \beta_{j} ; q\right] f\right)^{\prime \prime}(z)}{\left(\mathcal{N}_{r, s}^{n}\left[\alpha_{i}, \beta_{j} ; q\right] f\right)^{\prime}(z)}+2\right)\right|+\eta,
\end{gathered}
$$

where $\mathcal{N}_{r, s}^{n}\left[\alpha_{i}, \beta_{j} ; q\right] f$ is defined in (10) and $\alpha \geq 0,-1 \leq \eta<$ $1, \alpha+\eta \geq 0, b \in \mathbb{C} \backslash\{0\}$.

Definition 6. Let a function $f \in \Omega$ be analytic in $\mathbb{U}^{*}$. Then $f$ is in the class $\Omega_{r, s} \mathscr{K} \mathscr{U} \mathscr{H}\left(\alpha_{i}, \beta_{j}, q, \alpha, b\right)$ if, and only if, $f$ satisfies

$$
\begin{aligned}
\mid 1- & \frac{1}{b}\left(\frac{z\left(\mathcal{N}_{r, s}^{n}\left[\alpha_{i}, \beta_{j} ; q\right] f\right)^{\prime \prime}(z)}{\left(\mathcal{N}_{r, s}^{n}\left[\alpha_{i}, \beta_{j} ; q\right] f\right)^{\prime}(z)}+2\right)-2 \alpha(\sqrt{2}-1) \mid \\
< & \operatorname{Re}\left\{\sqrt{2}\left(1-\frac{1}{b}\left(\frac{z\left(\mathcal{N}_{r, s}^{n}\left[\alpha_{i}, \beta_{j} ; q\right] f\right)^{\prime \prime}(z)}{\left(\mathcal{N}_{r, s}^{n}\left[\alpha_{i}, \beta_{j} ; q\right] f\right)^{\prime}(z)}+2\right)\right)\right\} \\
& +2 \alpha(\sqrt{2}-1),
\end{aligned}
$$

where $\mathcal{N}_{r, s}^{n}\left[\alpha_{i}, \beta_{j} ; q\right] f$ is defined in (10) and $\alpha>0, b \in \mathbb{C} \backslash\{0\}$.

Remark 7. Putting $n=0, r=1, s=0$ and $\alpha_{1}=q$ in Definition 3, we obtain the result corresponding to the class mentioned in (3).

For $n=0, r=1, s=0$ and $\alpha_{1}=q$ in Definitions 4,5 , and 6, we obtain $\Omega \mathscr{K}_{b}(\eta), \Omega \mathscr{K} \mathscr{U}(\alpha, \eta, b)$ and $\Omega \mathscr{K} \mathscr{H}(\alpha, b)$ the classes of meromorphic functions, introduced and studied by Mohammed and Darus [20].

\section{Preliminary Definitions}

We begin by recalling each of the following definitions of subclasses of meromorphic functions $\Omega \mathscr{G}_{1}(\eta, b), \Omega \mathscr{G}_{2}(\alpha, \eta, b)$ and $\Omega \mathscr{G}_{3}(\alpha, b)$ which will be required in our investigation.

Definition 8 (see [20]). Let a function $f \in \Omega$ be analytic in $\mathbb{U}^{*}$. Then $f$ is in the class $\Omega \mathscr{G}_{1}(\eta, b)$ if, and only if, $f$ satisfies

$$
\mathfrak{R e}\left\{1-\frac{1}{b}\left(\frac{z\left(z f^{\prime \prime}(z)+3 f^{\prime}(z)\right)}{z f^{\prime}(z)+2 f(z)}+1\right)\right\}>\eta
$$

where $b \in \mathbb{C} \backslash\{0\}, 0 \leq \eta<1$.
Definition 9 (see [20]). Let a function $f \in \Omega$ be analytic in $\mathbb{U}^{*}$. Then $f$ is in the class $\Omega \mathscr{G}_{2}(\alpha, \eta, b)$ if, and only if, $f$ satisfies

$$
\begin{gathered}
\mathfrak{R e}\left\{1-\frac{1}{b}\left(\frac{z\left(z f^{\prime \prime}(z)+3 f^{\prime}(z)\right)}{z f^{\prime}(z)+2 f(z)}+1\right)\right\} \\
>\alpha\left|\frac{1}{b}\left(\frac{z\left(z f^{\prime \prime}(z)+3 f^{\prime}(z)\right)}{z f^{\prime}(z)+2 f(z)}+1\right)\right|+\eta,
\end{gathered}
$$

where $\alpha \geq 0,-1 \leq \eta<1, \alpha+\eta \geq 0, b \in \mathbb{C} \backslash\{0\}$.

Definition 10 (see [20]). Let a function $f \in \Omega$ be analytic in $\mathbb{U}^{*}$. Then $f$ is in the class $\Omega \mathscr{G}_{3}(\alpha, b)$ if, and only if, $f$ satisfies

$$
\begin{aligned}
\mid 1- & \frac{1}{b}\left(\frac{z\left(z f^{\prime \prime}(z)+3 f^{\prime}(z)\right)}{z f^{\prime}(z)+2 f(z)}+1\right)-2 \alpha(\sqrt{2}-1) \mid \\
< & \Re \mathfrak{R}\left\{\sqrt{2}\left(1-\frac{1}{b}\left(\frac{z\left(z f^{\prime \prime}(z)+3 f^{\prime}(z)\right)}{z f^{\prime}(z)+2 f(z)}+1\right)\right)\right\} \\
& +2 \alpha(\sqrt{2}-1),
\end{aligned}
$$

where $\alpha>0, b \in \mathbb{C} \backslash\{0\}$.

\section{Main Results}

In this section, we study some properties for the integral operator $l_{\delta_{1}, \ldots, \delta_{n}}(z)$ defined by (12) of the subclasses given by Definitions $3,4,5$, and 6 .

Theorem 11. Let $\delta_{i}>0$, for $i \in\{1,2, \ldots, n\}$ and $f_{i} \in \Omega$. If

$$
\sum_{i=1}^{n} \delta_{i}\left(1-\eta_{i}\right)<2, \quad\left(0 \leq \eta_{i}<1\right)
$$

and $f_{i} \in \Omega_{k}\left(\alpha_{i}, \beta_{j}, q, \eta_{i}\right)$, then $l_{\delta_{1}, \ldots, \delta_{n}}(z)$ is in the class $\Omega_{N}(\xi)$, $\xi>1$.

Proof. By differentiating the equality given by (12), we get

$$
\begin{aligned}
z^{2} l_{\delta_{1}, \ldots, \delta_{n}}^{\prime}(z)+2 z l_{\delta_{1}, \ldots, \delta_{n}}(z) \\
=\left(-z^{2}\left(\mathcal{N}_{r, s}^{n}\left[\alpha_{i}, \beta_{j} ; q\right] f_{1}\right)^{\prime}(z)\right)^{\delta_{1}} \\
\quad \ldots\left(-z^{2}\left(\mathcal{N}_{r, s}^{n}\left[\alpha_{i}, \beta_{j} ; q\right] f_{n}\right)^{\prime}(z)\right)^{\delta_{n}} .
\end{aligned}
$$

Again, differentiating (21), we deduce that

$$
\begin{aligned}
& z^{2} l_{\delta_{1}, \ldots, \delta_{n}}^{\prime \prime}(z)+4 z l_{\delta_{1}, \ldots, \delta_{n}}^{\prime}(z)+2 l_{\delta_{1}, \ldots, \delta_{n}}(z) \\
& =\sum_{i=1}^{n} \delta_{i}\left(\frac{\left(\mathcal{N}_{r, s}^{n}\left[\alpha_{i}, \beta_{j} ; q\right] f_{i}\right)^{\prime \prime}(z)}{\left(\mathcal{N}_{r, s}^{n}\left[\alpha_{i}, \beta_{j} ; q\right] f_{i}\right)^{\prime}(z)}+\frac{2}{z}\right), \\
& {\left[\left(-z^{2}\left(\mathcal{N}_{r, s}^{n}\left[\alpha_{i}, \beta_{j} ; q\right] f_{1}\right)^{\prime}(z)\right)^{\delta_{1}}\right.} \\
& \left.\ldots\left(-z^{2}\left(\mathcal{N}_{r, s}^{n}\left[\alpha_{i}, \beta_{j} ; q\right] f_{n}\right)^{\prime}(z)\right)^{\delta_{n}}\right] .
\end{aligned}
$$


By using (21) and (23), we have

$$
\begin{gathered}
\frac{z^{2} l_{\delta_{1}, \ldots, \delta_{n}}^{\prime \prime}(z)+4 z l_{\delta_{1}, \ldots, \delta_{n}}^{\prime}(z)+2 l_{\delta_{1}, \ldots, \delta_{n}}(z)}{z^{2} l_{\delta_{1}, \ldots, \delta_{n}}^{\prime}(z)+2 z l_{\delta_{1}, \ldots, \delta_{n}}(z)} \\
=\sum_{i=1}^{n} \delta_{i}\left(\frac{\left(\mathcal{N}_{r, s}^{n}\left[\alpha_{i}, \beta_{j} ; q\right] f_{i}\right)^{\prime \prime}(z)}{\left(\mathcal{N}_{r, s}^{n}\left[\alpha_{i}, \beta_{j} ; q\right] f_{i}\right)^{\prime}(z)}+\frac{2}{z}\right) .
\end{gathered}
$$

Multiplying (24) by $z$ yields that

$$
\begin{gathered}
\frac{z^{2} l_{\delta_{1}, \ldots, \delta_{n}}^{\prime \prime}(z)+4 z l_{\delta_{1}, \ldots, \delta_{n}}^{\prime}(z)+2 l_{\delta_{1}, \ldots, \delta_{n}}(z)}{z l_{\delta_{1}, \ldots, \delta_{n}}^{\prime}(z)+2 l_{\delta_{1}, \ldots, \delta_{n}}(z)} \\
=\sum_{i=1}^{n} \delta_{i}\left(\frac{z\left(\mathcal{N}_{r, s}^{n}\left[\alpha_{i}, \beta_{j} ; q\right] f_{i}\right)^{\prime \prime}(z)}{\left(\mathcal{N}_{r, s}^{n}\left[\alpha_{i}, \beta_{j} ; q\right] f_{i}\right)^{\prime}(z)}+2\right),
\end{gathered}
$$

or, equivalently, that

$$
\begin{aligned}
& \frac{z\left(z l_{\delta_{1}, \ldots, \delta_{n}}^{\prime \prime}(z)+3 l_{\delta_{1}, \ldots, \delta_{n}}^{\prime}(z)\right)}{z l_{\delta_{1}, \ldots, \delta_{n}}^{\prime}(z)+2 l_{\delta_{1}, \ldots, \delta_{n}}(z)}+1 \\
& \quad=\sum_{i=1}^{n} \delta_{i}\left(\frac{z\left(\mathcal{N}_{r, s}^{n}\left[\alpha_{i}, \beta_{j} ; q\right] f_{i}\right)^{\prime \prime}(z)}{\left(\mathcal{N}_{r, s}^{n}\left[\alpha_{i}, \beta_{j} ; q\right] f_{i}\right)^{\prime}(z)}+2\right) .
\end{aligned}
$$

Thus, we get

$$
\begin{aligned}
& -\left(\frac{z l_{\delta_{1}, \ldots, \delta_{n}}^{\prime \prime}(z)}{l_{\delta_{1}, \ldots, \delta_{n}}^{\prime}(z)}+1\right)-2 \\
& =\left(-\sum_{i=1}^{n} \delta_{i}\left(\frac{z\left(\mathcal{N}_{r, s}^{n}\left[\alpha_{i}, \beta_{j} ; q\right] f_{i}\right)^{\prime \prime}(z)}{\left(\mathcal{N}_{r, s}^{n}\left[\alpha_{i}, \beta_{j} ; q\right] f_{i}\right)^{\prime}(z)}+2\right)+1\right) \\
& \quad \times\left(1+\frac{2 l_{\delta_{1}, \ldots, \delta_{n}}(z)}{z l_{\delta_{1}, \ldots, \delta_{n}}^{\prime}(z)}\right) .
\end{aligned}
$$

That is,

$$
\begin{aligned}
& -\left(\frac{z l_{\delta_{1}, \ldots, \delta_{n}}^{\prime \prime}(z)}{l_{\delta_{1}, \ldots, \delta_{n}}^{\prime}(z)}+1\right) \\
& =\left(\frac{2 l_{\delta_{1}, \ldots, \delta_{n}}(z)}{z l_{\delta_{1}, \ldots, \delta_{n}}^{\prime}(z)}\right) \\
& \quad \times\left(-\sum_{i=1}^{n} \delta_{i}\left(\frac{z\left(\mathcal{N}_{r, s}^{n}\left[\alpha_{i}, \beta_{j} ; q\right] f_{i}\right)^{\prime \prime}(z)}{\left(\mathcal{N}_{r, s}^{n}\left[\alpha_{i}, \beta_{j} ; q\right] f_{i}\right)^{\prime}(z)}+2\right)+1\right) \\
& \quad+\sum_{i=1}^{n} \delta_{i}\left(-\left(\frac{z\left(\mathcal{N}_{r, s}^{n}\left[\alpha_{i}, \beta_{j} ; q\right] f_{i}\right)^{\prime \prime}(z)}{\left(\mathcal{N}_{r, s}^{n}\left[\alpha_{i}, \beta_{j} ; q\right] f_{i}\right)^{\prime}(z)}+1\right)\right) \\
& \quad+3-\sum_{i=1}^{n} \delta_{i} .
\end{aligned}
$$

Taking the real parts of both terms of the last expression and using the fact that $\operatorname{Re}(z) \leq|z|$ for all $z$, we impose

$$
\begin{aligned}
-\operatorname{Re}\left(\frac{z l_{\delta_{1}, \ldots, \delta_{n}}^{\prime \prime}(z)}{l_{\delta_{1}, \ldots, \delta_{n}}^{\prime}(z)}+1\right) & \operatorname{Re}\left\{\left(\frac{2 l_{\delta_{1}, \ldots, \delta_{n}}(z)}{z l_{\delta_{1}, \ldots, \delta_{n}}^{\prime}(z)}\right)\right. \\
+ & \left.\times\left(-\sum_{i=1}^{n} \delta_{i}\left(\frac{z\left(\mathcal{N}_{r, s}^{n}\left[\alpha_{i}, \beta_{j} ; q\right] f_{i}\right)^{\prime \prime}(z)}{\left(\mathcal{N}_{r, s}^{n}\left[\alpha_{i}, \beta_{j} ; q\right] f_{i}\right)^{\prime}(z)}+2\right)+1\right)\right\} \\
+ & \sum_{i=1}^{n} \delta_{i} \Re_{e}\left\{-\left(\frac{z\left(\mathcal{N}_{r, s}^{n}\left[\alpha_{i}, \beta_{j} ; q\right] f_{i}\right)^{\prime \prime}(z)}{\left(\mathcal{N}_{r, s}^{n}\left[\alpha_{i}, \beta_{j} ; q\right] f_{i}\right)^{\prime}(z)}+1\right)\right\} \\
+ & \left.\left.-\sum_{i=1}^{n} \delta_{i}\right)\right\}
\end{aligned}
$$$$
\leq \mid \frac{2 l_{\delta_{1}, \ldots, \delta_{n}}(z)}{z l_{\delta_{1}, \ldots, \delta_{n}}^{\prime}(z)}
$$

$$
\begin{aligned}
& \times\left(-\sum_{i=1}^{n} \delta_{i}\left(\frac{z\left(\mathcal{N}_{r, s}^{n}\left[\alpha_{i}, \beta_{j} ; q\right] f_{i}\right)^{\prime \prime}(z)}{\left(\mathcal{N}_{r, s}^{n}\left[\alpha_{i}, \beta_{j}, q\right] f_{i}\right)^{\prime}(z)}+2\right)+1\right) \mid \\
+ & \sum_{i=1}^{n} \delta_{i} \mathfrak{R e}\left\{-\left(\frac{z\left(\mathcal{N}_{r, s}^{n}\left[\alpha_{i}, \beta_{j}, q\right] f_{i}\right)^{\prime \prime}(z)}{\left(\mathcal{N}_{r, s}^{n}\left[\alpha_{i}, \beta_{j} ; q\right] f_{i}\right)^{\prime}(z)}+1\right)\right\} \\
+ & 3-\sum_{i=1}^{n} \delta_{i} .
\end{aligned}
$$

Setting

$$
\begin{aligned}
\xi=\mid \frac{2 l_{\delta_{1}, \ldots, \delta_{n}}(z)}{z l_{\delta_{1}, \ldots, \delta_{n}}^{\prime}(z)} \\
\quad \times\left(-\sum_{i=1}^{n} \delta_{i}\left(\frac{z\left(\mathcal{N}_{r, s}^{n}\left[\alpha_{i}, \beta_{j} ; q\right] f_{i}\right)^{\prime \prime}(z)}{\left(\mathcal{N}_{r, s}^{n}\left[\alpha_{i}, \beta_{j} ; q\right] f_{i}\right)^{\prime}(z)}+2\right)+1\right) \mid \\
+\sum_{i=1}^{n} \delta_{i} \mathfrak{R e}\left(-\left(\frac{z\left(\mathcal{N}_{r, s}^{n}\left[\alpha_{i}, \beta_{j} ; q\right] f_{i}\right)^{\prime \prime}(z)}{\left(\mathcal{N}_{r, s}^{n}\left[\alpha_{i}, \beta_{j} ; q\right] f_{i}\right)^{\prime}(z)}+1\right)\right) \\
+3-\sum_{i=1}^{n} \delta_{i},
\end{aligned}
$$


and since

$$
\begin{aligned}
& \mid \frac{2 l_{\delta_{1}, \ldots, \delta_{n}}(z)}{z l_{\delta_{1}, \ldots, \delta_{n}}^{\prime}(z)} \\
& \quad \times\left(-\sum_{i=1}^{n} \delta_{i}\left(\frac{z\left(\mathcal{N}_{r, s}^{n}\left[\alpha_{i}, \beta_{j} ; q\right] f_{i}\right)^{\prime \prime}(z)}{\left(\mathcal{N}_{r, s}^{n}\left[\alpha_{i}, \beta_{j} ; q\right] f_{i}\right)^{\prime}(z)}+2\right)+1\right) \mid \\
& \quad>0, \quad f_{i} \in \Omega_{k}\left(\alpha_{i}, \beta_{j}, q, \eta_{i}\right),
\end{aligned}
$$

we conclude that

$$
\xi>\sum_{i=1}^{n} \delta_{i}\left(\eta_{i}-1\right)+3
$$

Hence, by (20), we have $\xi>1$, so that $l_{\delta_{1}, \ldots, \delta_{n}}(z) \in \Omega_{N}(\xi)$, which evidently completes the proof of Theorem 11.

Theorem 12. Let $\delta_{i}>0$, for $i \in\{1,2, \ldots, n\}, f_{i} \in$ $\Omega_{r, s} \mathscr{K}\left(\alpha_{i}, \beta_{j}, q, \eta_{i}, b\right)\left(0 \leq \eta_{i}<1\right)$ and $b \in \mathbb{C} \backslash\{0\}$. If

$$
0<\sum_{i=1}^{n} \delta_{i}\left(1-\eta_{i}\right) \leq 1
$$

then $l_{\delta_{1}, \ldots, \delta_{n}}(z)$ is in the class $\Sigma \mathscr{G}_{1}(\gamma, b)$, where $\gamma=1-$ $\sum_{i=1}^{n} \delta_{i}\left(1-\eta_{i}\right)$.

Proof. We know from the proof of Theorem 11 that

$$
\begin{aligned}
& \frac{z\left(z l_{\delta_{1}, \ldots, \delta_{n}}^{\prime \prime}(z)+3 l_{\delta_{1}, \ldots, \delta_{n}}^{\prime}(z)\right)}{z l_{\delta_{1}, \ldots, \delta_{n}}^{\prime}(z)+2 l_{\delta_{1}, \ldots, \delta_{n}}(z)}+1 \\
& \quad=\sum_{i=1}^{n} \delta_{i}\left(\frac{z\left(\mathcal{N}_{r, s}^{n}\left[\alpha_{i}, \beta_{j} ; q\right] f_{i}\right)^{\prime \prime}(z)}{\left(\mathcal{N}_{r, s}^{n}\left[\alpha_{i}, \beta_{j} ; q\right] f_{i}\right)^{\prime}(z)}+2\right),
\end{aligned}
$$

which is equivalent to

$$
\begin{aligned}
1 & -\frac{1}{b}\left\{\frac{z\left(z l_{\delta_{1}, \ldots, \delta_{n}}^{\prime \prime}(z)+3 l_{\delta_{1}, \ldots, \delta_{n}}^{\prime}(z)\right)}{z l_{\delta_{1}, \ldots, \delta_{n}}^{\prime}(z)+2 l(z)}+1\right\} \\
= & 1-\sum_{i=1}^{n} \delta_{i} \\
& +\sum_{i=1}^{n} \delta_{i}\left\{1-\frac{1}{b}\left(\frac{z\left(\mathcal{N}_{r, s}^{n}\left[\alpha_{i}, \beta_{j} ; q\right] f_{i}\right)^{\prime \prime}(z)}{\left(\mathscr{N}_{r, s}^{n}\left[\alpha_{i}, \beta_{j} ; q\right] f_{i}\right)^{\prime}(z)}+2\right)\right\} .
\end{aligned}
$$

Taking the real part of both terms of (35), we get

$$
\begin{aligned}
\mathfrak{R e} & \left\{1-\frac{1}{b}\left(\frac{z\left(z l_{\delta_{1}, \ldots, \delta_{n}}^{\prime \prime}(z)+3 l_{\delta_{1}, \ldots, \delta_{n}}^{\prime}(z)\right)}{z l_{\delta_{1}, \ldots, \delta_{n}}^{\prime}(z)+2 l_{\delta_{1}, \ldots, \delta_{n}}(z)}+1\right)\right\} \\
= & \sum_{i=1}^{n} \delta_{i} \mathfrak{R} \mathfrak{e}\left\{1-\frac{1}{b}\left(\frac{z\left(\mathcal{N}_{r, s}^{n}\left[\alpha_{i}, \beta_{j} ; q\right] f_{i}\right)^{\prime \prime}(z)}{\left(\mathcal{N}_{r, s}^{n}\left[\alpha_{i}, \beta_{j} ; q\right] f_{i}\right)^{\prime}(z)}+2\right)\right\} \\
& +1-\sum_{i=1}^{n} \delta_{i} .
\end{aligned}
$$

Now, since $f_{i} \in \Omega_{r, s} \mathscr{K}\left(\alpha_{i}, \beta_{j}, q, \eta_{i}, b\right)$, we have

$$
\begin{gathered}
\mathfrak{R e}\left\{1-\frac{1}{b}\left(\frac{z\left(z l_{\delta_{1}, \ldots, \delta_{n}}^{\prime \prime}(z)+3 l_{\delta_{1}, \ldots, \delta_{n}}^{\prime}(z)\right)}{z l_{\delta_{1}, \ldots, \delta_{n}}^{\prime \prime}(z)+2 l_{\delta_{1}, \ldots, \delta_{n}}(z)}+1\right)\right\} \\
>\sum_{i=1}^{n} \delta_{i} \eta_{i}+1-\sum_{i=1}^{n} \delta_{i}=1-\sum_{i=1}^{n} \delta_{i}\left(1-\eta_{i}\right) .
\end{gathered}
$$

Letting

$$
\gamma=1-\sum_{i=1}^{n} \delta_{i}\left(1-\eta_{i}\right)
$$

and in view of (33), it follows that $0 \leq \gamma<1$, so that $l_{\delta_{1}, \ldots, \delta_{n}}(z) \in \Omega \mathscr{G}_{1}(\gamma, b)$. This completes the proof of Theorem 12 .

Theorem 13. Let $\delta_{i}>0$, for $i \in\{1,2, \ldots, n\}, f_{i} \in$ $\Omega_{r, s} \mathscr{K} \mathscr{U}\left(\alpha_{i}, \beta_{j}, q, \alpha, \eta, b\right)(\alpha \geq 0,-1 \leq \eta<1, \alpha+\eta \geq 0)$ and $b \in \mathbb{C} \backslash\{0\}$. If

$$
\sum_{i=1}^{n} \delta_{i}-1 \leq 0
$$

then $l_{\delta_{1}, \ldots, \delta_{n}}(z)$ is in the class $\Omega \mathscr{G}_{2}(\alpha, \eta, b)$.

Proof. We want to show that

$$
\begin{aligned}
\mathfrak{R e} & \left\{1-\frac{1}{b}\left(\frac{z\left(z l_{\delta_{1}, \ldots, \delta_{n}}^{\prime \prime}(z)+3 l_{\delta_{1}, \ldots, \delta_{n}}^{\prime}(z)\right)}{z l_{\delta_{1}, \ldots, \delta_{n}}^{\prime \prime}(z)+2 l_{\delta_{1}, \ldots, \delta_{n}}(z)}+1\right)\right\} \\
& -\alpha\left|\frac{1}{b}\left(\frac{z\left(z l_{\delta_{1}, \ldots, \delta_{n}}^{\prime \prime}(z)+3 l_{\delta_{1}, \ldots, \delta_{n}}^{\prime}(z)\right)}{z l_{\delta_{1}, \ldots, \delta_{n}}^{\prime}(z)+2 l_{\delta_{1}, \ldots, \delta_{n}}(z)}+1\right)\right|-\eta
\end{aligned}
$$


We find from (26) and (36) that

$$
\begin{aligned}
\mathfrak{R e}\{1 & \left.-\frac{1}{b}\left(\frac{z\left(z l_{\delta_{1}, \ldots, \delta_{n}}^{\prime \prime}(z)+3 l_{\delta_{1}, \ldots, \delta_{n}}^{\prime}(z)\right)}{z l_{\delta_{1}, \ldots, \delta_{n}}^{\prime}(z)+2 l_{\delta_{1}, \ldots, \delta_{n}}(z)}+1\right)\right\} \\
& -\alpha\left|\frac{1}{b}\left(\frac{z\left(z l_{\delta_{1}, \ldots, \delta_{n}}^{\prime \prime}(z)+3 l^{\prime}(z)\right)}{z l_{\delta_{1}, \ldots, \delta_{n}}^{\prime}(z)+2 l_{\delta_{1}, \ldots, \delta_{n}}(z)}+1\right)\right|-\eta \\
= & -\sum_{i=1}^{n} \delta_{i} \\
& +\sum_{i=1}^{n} \delta_{i} \mathfrak{R e}\left\{1-\frac{1}{b}\left(\frac{z\left(\mathcal{N}_{r, s}^{n}\left[\alpha_{i}, \beta_{j} ; q\right] f_{i}\right)^{\prime \prime}(z)}{\left(\mathcal{N}_{r, s}^{n}\left[\alpha_{i}, \beta_{j} ; q\right] f_{i}\right)^{\prime}(z)}+2\right)\right\} \\
& -\alpha\left|\sum_{i=1}^{n} \delta_{i} \frac{1}{b}\left(\frac{z\left(\mathcal{N}_{r, s}^{n}\left[\alpha_{i}, \beta_{j} ; q\right] f_{i}\right)^{\prime \prime}(z)}{\left(\mathcal{N}_{r, s}^{n}\left[\alpha_{i}, \beta_{j} ; q\right] f_{i}\right)^{\prime}(z)}+2\right)\right|-\eta .
\end{aligned}
$$

Since $f_{i} \in \Omega_{r, s} \mathscr{K} \mathscr{U}\left(\alpha_{i}, \beta_{j}, q, \alpha, \delta, b\right)$, it follows from Definition 5 that

$$
\begin{aligned}
\mathfrak{R e}\left\{1-\frac{1}{b}\left(\frac{z\left(z l_{\delta_{1}, \ldots, \delta_{n}}^{\prime \prime}(z)+3 l_{\delta_{1}, \ldots, \delta_{n}}^{\prime}(z)\right)}{z l_{\delta_{1}, \ldots, \delta_{n}}^{\prime}(z)+2 l_{\delta_{1}, \ldots, \delta_{n}}(z)}+1\right)\right\} \\
-\alpha\left|\frac{1}{b}\left(\frac{z\left(z l_{\delta_{1}, \ldots, \delta_{n}}^{\prime \prime}(z)+3 l_{\delta_{1}, \ldots, \delta_{n}}^{\prime}(z)\right)}{z l_{\delta_{1}, \ldots, \delta_{n}}^{\prime}(z)+2 l_{\delta_{1}, \ldots, \delta_{n}}(z)}+1\right)\right|-\eta \\
>1-\sum_{i=1}^{n} \delta_{i} \\
+\sum_{i=1}^{n} \delta_{i}\left\{\alpha\left|\frac{1}{b}\left(\frac{z\left(\mathcal{N}_{r, s}^{n}\left[\alpha_{i}, \beta_{j} ; q\right] f_{i}\right)^{\prime \prime}(z)}{\left(\mathcal{N}_{r, s}^{n}\left[\alpha_{i}, \beta_{j} ; q\right] f_{i}\right)^{\prime}(z)}+2\right)\right|+\eta\right\} \\
\quad-\alpha \sum_{i=1}^{n} \delta_{i}\left|\frac{1}{b}\left(\frac{z\left(\mathcal{N}_{r, s}^{n}\left[\alpha_{i}, \beta_{j} ; q\right] f_{i}\right)^{\prime \prime}(z)}{\left(\mathcal{N}_{r, s}^{n}\left[\alpha_{i}, \beta_{j} ; q\right] f_{i}\right)^{\prime}(z)}+2\right)\right|-\eta \\
=1-\sum_{i=1}^{n} \delta_{i}+\sum_{i=1}^{n} \delta_{i} \eta-\eta \cdot
\end{aligned}
$$

From hypothesis (39), we note that

$$
(1-\eta)\left(1-\sum_{i=1}^{n} \delta_{i}\right) \geq 0 .
$$

Hence $l_{\delta_{1}, \ldots, \delta_{n}} \in \Omega \mathscr{G}_{2}(\alpha, \eta, b)$. Therefore, we complete the proof of Theorem 13 .

Theorem 14. Let $\delta_{i}>0$ for $i \in\{1,2, \ldots, n\}, f_{i} \in$ $\Omega_{r, s} \mathscr{K} \mathscr{H}\left(\alpha_{i}, \beta_{j}, q ; \alpha, b\right)(\alpha>0$ and $b \in \mathbb{C} \backslash\{0\})$. If

$$
\sum_{i=1}^{n} \delta_{i}-1 \leq 0
$$

then $l_{\delta_{1}, \ldots, \delta_{n}}(z)$ is in the class $\Omega \mathscr{G}_{3}(\alpha, b)$.
Proof. Our aim is to prove that

$$
\begin{aligned}
& \mathfrak{R}\left\{\sqrt{2}\left(1-\frac{1}{b}\left(\frac{z\left(z l_{\delta_{1}, \ldots, \delta_{n}}^{\prime \prime}(z)+3 l_{\delta_{1}, \ldots, \delta_{n}}^{\prime}(z)\right)}{z l_{\delta_{1}, \ldots, \delta_{n}}^{\prime}(z)+2 l_{\delta_{1}, \ldots, \delta_{n}}(z)}+1\right)\right)\right\} \\
& +2 \alpha(\sqrt{2}-1) \\
& -\mid 1-\frac{1}{b}\left(\frac{z\left(z l_{\delta_{1}, \ldots, \delta_{n}}^{\prime \prime}(z)+3 l_{\delta_{1}, \ldots, \delta_{n}}^{\prime}(z)\right)}{z l_{\delta_{1}, \ldots, \delta_{n}}^{\prime}(z)+2 l_{\delta_{1}, \ldots, \delta_{n}}(z)}+1\right) \\
& -2 \alpha(\sqrt{2}-1) \\
& >0 \text {. }
\end{aligned}
$$

With the aid of (26), (36) can be written as follows:

$$
\begin{aligned}
& \mathfrak{R}\left\{\sqrt{2}\left(1-\frac{1}{b}\left(\frac{z\left(z l_{\delta_{1}, \ldots, \delta_{n}}^{\prime \prime}(z)+3 l_{\delta_{1}, \ldots, \delta_{n}}^{\prime}(z)\right)}{z l_{\delta_{1}, \ldots, \delta_{n}}^{\prime}(z)+2 l_{\delta_{1}, \ldots, \delta_{n}}(z)}+1\right)\right)\right\} \\
& +2 \alpha(\sqrt{2}-1) \\
& -\mid 1-\frac{1}{b}\left(\frac{z\left(z l_{\delta_{1}, \ldots, \delta_{n}}^{\prime \prime}(z)+3 l_{\delta_{1}, \ldots, \delta_{n}}^{\prime}(z)\right)}{z l_{\delta_{1}, \ldots, \delta_{n}}^{\prime}(z)+2 l_{\delta_{1}, \ldots, \delta_{n}}(z)}+1\right) \\
& -2 \alpha(\sqrt{2}-1) \\
& =\Re\left\{\sqrt { 2 } \left[1-\sum_{i=1}^{n} \delta_{i} \frac{1}{b}\right.\right. \\
& \left.\left.\times\left(\frac{z\left(\mathcal{N}_{r, s}^{n}\left[\alpha_{i}, \beta_{j} ; q\right] f_{i}\right)^{\prime \prime}(z)}{\left(\mathcal{N}_{r, s}^{n}\left[\alpha_{i}, \beta_{j} ; q\right] f_{i}\right)^{\prime}(z)}+2\right)\right]\right\} \\
& +2 \alpha(\sqrt{2}-1) \\
& -\mid 1-\sum_{i=1}^{n} \delta_{i} \frac{1}{b}\left(\frac{z\left(\mathcal{N}_{r, s}^{n}\left[\alpha_{i}, \beta_{j} ; q\right] f_{i}\right)^{\prime \prime}(z)}{\left(\mathcal{N}_{r, s}^{n}\left[\alpha_{i}, \beta_{j} ; q\right] f_{i}\right)^{\prime}(z)}+2\right) \\
& -2 \alpha(\sqrt{2}-1) \\
& =\sqrt{2}-\sqrt{2} \sum_{i=1}^{n} \delta_{i} \Re \frac{1}{b}\left(\frac{z\left(\mathcal{N}_{r, s}^{n}\left[\alpha_{i}, \beta_{j} ; q\right] f_{i}\right)^{\prime \prime}(z)}{\left(\mathcal{N}_{r, s}^{n}\left[\alpha_{i}, \beta_{j} ; q\right] f_{i}\right)^{\prime}(z)}+2\right) \\
& +2 \alpha(\sqrt{2}-1)
\end{aligned}
$$




$$
\begin{aligned}
& -\mid 1-\sum_{i=1}^{n} \delta_{i} \frac{1}{b}\left(\frac{z\left(\mathcal{N}_{r, s}^{n}\left[\alpha_{i}, \beta_{j} ; q\right] f_{i}\right)^{\prime \prime}(z)}{\left(\mathcal{N}_{r, s}^{n}\left[\alpha_{i}, \beta_{j} ; q\right] f_{i}\right)^{\prime}(z)}+2\right) \\
& -2 \alpha(\sqrt{2}-1) \mid \\
& =\sqrt{2}+\sqrt{2} \sum_{i=1}^{n} \delta_{i} \mathfrak{R} \\
& \times\left\{1-\frac{1}{b}\left(\frac{z\left(\mathcal{N}_{r, s}^{n}\left[\alpha_{i}, \beta_{j} ; q\right] f_{i}\right)^{\prime \prime}(z)}{\left(\mathcal{N}_{r, s}^{n}\left[\alpha_{i}, \beta_{j} ; q\right] f_{i}\right)^{\prime}(z)}+2\right)\right\} \\
& -\sqrt{2} \sum_{i=1}^{n} \delta_{i}+2 \alpha(\sqrt{2}-1) \\
& -\mid 1+\sum_{i=1}^{n} \delta_{i}\left[1-\frac{1}{b}\left(\frac{z\left(\mathcal{N}_{r, s}^{n}\left[\alpha_{i}, \beta_{j} ; q\right] f_{i}\right)^{\prime \prime}(z)}{\left(\mathcal{N}_{r, s}^{n}\left[\alpha_{i}, \beta_{j} ; q\right] f_{i}\right)^{\prime}(z)}+2\right)\right. \\
& -2 \alpha(\sqrt{2}-1)] \\
& -\sum_{i=1}^{n} \delta_{i}+2 \alpha(\sqrt{2}-1) \sum_{i=1}^{n} \delta_{i}-2 \alpha(\sqrt{2}-1) \\
& =\sqrt{2}\left(1-\sum_{i=1}^{n} \delta_{i}\right)+2 \alpha(\sqrt{2}-1) \\
& +\sqrt{2} \sum_{i=1}^{n} \delta_{i} \mathfrak{R} \\
& \times\left\{1-\frac{1}{b}\left(\frac{z\left(\mathcal{N}_{r, s}^{n}\left[\alpha_{i}, \beta_{j} ; q\right] f_{i}\right)^{\prime \prime}(z)}{\left(\mathcal{N}_{r, s}^{n}\left[\alpha_{i}, \beta_{j} ; q\right] f_{i}\right)^{\prime}(z)}+2\right)\right\} \\
& -\mid[1-2 \alpha(\sqrt{2}-1)]\left(1-\sum_{i=1}^{n} \delta_{i}\right) \\
& +\sum_{i=1}^{n} \delta_{i}\left[1-\frac{1}{b}\left(\frac{z\left(\mathcal{N}_{r, s}^{n}\left[\alpha_{i}, \beta_{j} ; q\right] f_{i}\right)^{\prime \prime}(z)}{\left(\mathcal{N}_{r, s}^{n}\left[\alpha_{i}, \beta_{j} ; q\right] f_{i}\right)^{\prime}(z)}+2\right)\right. \\
& -2 \alpha(\sqrt{2}-1)] \mid \\
& \geq \sqrt{2}\left(1-\sum_{i=1}^{n} \delta_{i}\right)+2 \alpha(\sqrt{2}-1) \\
& +\sqrt{2} \sum_{i=1}^{n} \delta_{i} \mathfrak{R}
\end{aligned}
$$$$
\times\left\{1-\frac{1}{b}\left(\frac{z\left(\mathcal{N}_{r, s}^{n}\left[\alpha_{i}, \beta_{j} ; q\right] f_{i}\right)^{\prime \prime}(z)}{\left(\mathcal{N}_{r, s}^{n}\left[\alpha_{i}, \beta_{j} ; q\right] f_{i}\right)^{\prime}(z)}+2\right)\right\}
$$$$
-\sum_{i=1}^{n} \delta_{i} \mid 1-\frac{1}{b}\left(\frac{z\left(\mathcal{N}_{r, s}^{n}\left[\alpha_{i}, \beta_{j} ; q\right] f_{i}\right)^{\prime \prime}(z)}{\left(\mathcal{N}_{r, s}^{n}\left[\alpha_{i}, \beta_{j} ; q\right] f_{i}\right)^{\prime}(z)}+2\right)
$$$$
-2 \alpha(\sqrt{2}-1)
$$$$
-|1-2 \alpha(\sqrt{2}-1)|\left(1-\sum_{i=1}^{n} \delta_{i}\right)
$$$$
=\sum_{i=1}^{n} \delta_{i}\{\Re \mathfrak{R} \sqrt{2}
$$$$
\times\left[1-\frac{1}{b}\left(\frac{z\left(\mathcal{N}_{r, s}^{n}\left[\alpha_{i}, \beta_{j} ; q\right] f_{i}\right)^{\prime \prime}(z)}{\left(\mathcal{N}_{r, s}^{n}\left[\alpha_{i}, \beta_{j} ; q\right] f_{i}\right)^{\prime}(z)}+2\right)\right]
$$$$
+2 \alpha(\sqrt{2}-1)
$$$$
-\mid 1-\frac{1}{b}\left(\frac{z\left(\mathscr{N}_{r, s}^{n}\left[\alpha_{i}, \beta_{j} ; q\right] f_{i}\right)^{\prime \prime}(z)}{\left(\mathcal{N}_{r, s}^{n}\left[\alpha_{i}, \beta_{j} ; q\right] f_{i}\right)^{\prime}(z)}+2\right)
$$$$
-2 \alpha(\sqrt{2}-1) \mid\}+\sqrt{2}\left(1-\sum_{i=1}^{n} \delta_{i}\right)
$$$$
+2 \alpha(\sqrt{2}-1)-2 \alpha(\sqrt{2}-1) \sum_{i=1}^{n} \delta_{i}
$$$$
-|1-2 \alpha(\sqrt{2}-1)|\left(1-\sum_{i=1}^{n} \delta_{i}\right) .
$$

Since $f_{i} \in \Omega_{r, s} \mathscr{K} \mathscr{H}\left(\alpha_{i}, \beta_{j}, q, \alpha, b\right)$, it follows from Definition 6 and by a simple calculation that

$$
\begin{aligned}
\Re\{ & \left.\sqrt{2}\left(1-\frac{1}{b}\left(\frac{z\left(z l_{\delta_{1}, \ldots, \delta_{n}}^{\prime \prime}(z)+3 l_{\delta_{1}, \ldots, \delta_{n}}^{\prime}(z)\right)}{z l_{\delta_{1}, \ldots, \delta_{n}}^{\prime}(z)+2 l_{\delta_{1}, \ldots, \delta_{n}}(z)}+1\right)\right)\right\} \\
+ & 2 \alpha(\sqrt{2}-1) \\
- & \mid 1-\frac{1}{b}\left(\frac{z\left(z l_{\delta_{1}, \ldots, \delta_{n}}^{\prime \prime}(z)+3 l_{\delta_{1}, \ldots, \delta_{n}}^{\prime}(z)\right)}{z l_{\delta_{1}, \ldots, \delta_{n}}^{\prime}(z)+2 l_{\delta_{1}, \ldots, \delta_{n}}(z)}+1\right) \\
& -2 \alpha(\sqrt{2}-1) \mid
\end{aligned}
$$




$$
\begin{aligned}
& >[\sqrt{2}+2 \alpha(\sqrt{2}-1)-|1-2 \alpha(\sqrt{2}-1)|]\left(1-\sum_{i=1}^{n} \delta_{i}\right) \\
& >\left(1-\sum_{i=1}^{n} \delta_{i}\right) \min \{(\sqrt{2}-1)(1+4 \alpha), \sqrt{2}+1\} \\
& \geq 0
\end{aligned}
$$

which means that $l_{\delta_{1}, \ldots, \delta_{n}} \in \Omega \mathscr{G}_{3}(\alpha, b)$, and we obtain the proof of the theorem.

\section{Conflict of Interests}

The authors declare that they have no competing interests.

\section{Authors' Contribution}

Ibtisam Aldawish and Maslina Darus read and approved the final paper.

\section{Acknowledgment}

The work presented here was partially supported by UKM's Grant FRGSTOPDOWN/2013/ST06/UKM/01/1.

\section{References}

[1] Z.-G. Wang, Y. Sun, and Z.-H. Zhang, "Certain classes of meromorphic multivalent functions," Computers \& Mathematics with Applications, vol. 58, no. 7, pp. 1408-1417, 2009.

[2] Z. Nehari and E. Netanyahu, "On the coefficients of meromorphic schlicht functions," Proceedings of the American Mathematical Society, vol. 8, pp. 15-23, 1957.

[3] G. Gasper and M. Rahman, Basic Hypergeometric Series, vol. 35 of Encyclopedia of Mathematics and Its Applications, Cambridge University Press, Cambridge, UK, 1990.

[4] H. Exton, \$q-Hypergeometric Functions and Applications, Ellis Horwood, Chichester, UK, 1983.

[5] H. A. Ghany, "q-derivative of basic hypergeometric series with respect to parameters," International Journal of Mathematical Analysis, vol. 3, no. 33, pp. 1617-1632, 2009.

[6] R. W. Ibrahim, "On certain linear operator defined by basic hypergeometric functions," Matematichki Vesnik, vol. 65, no. 1, pp. 1-7, 2013.

[7] R. W. Ibrahim and M. Darus, "On analytic functions associated with the Dziok-SRIvastava linear operator and Srivastava-Owa fractional integral operator," Arabian Journal for Science and Engineering, vol. 36, no. 3, pp. 441-450, 2011.

[8] I. Aldawish and M. Darus, "New subclass of analytic function associated with the generalized hypergeometric functions," Electronic Journal of Mathematical Analysis and Applications, vol. 2, no. 2, pp. 163-171, 2014.

[9] H. Aldweby and M. Darus, "Properties of a subclass of analytic functions defined by a generalized operator involving qHypergeometric function," Far East Journal of Mathematical Sciences, vol. 81, no. 2, pp. 189-200, 2013.

[10] B. A. Frasin and M. Darus, "On certain meromorphic functions with positive coefficients," Southeast Asian Bulletin of Mathematics, vol. 28, no. 4, pp. 615-623, 2004.
[11] R. M. El-Ashwah and M. K. Aouf, "Hadamard product of certain meromorphic starlike and convex functions," Computers \& Mathematics with Applications, vol. 57, no. 7, pp. 1102-1106, 2009.

[12] J.-L. Liu and H. M. Srivastava, "Classes of meromorphically multivalent functions associated with the generalized hypergeometric function," Mathematical and Computer Modelling, vol. 39, no. 1, pp. 21-34, 2004.

[13] J.-L. Liu and H. M. Srivastava, "A linear operator and associated families of meromorphically multivalent functions," Journal of Mathematical Analysis and Applications, vol. 259, no. 2, pp. 566581, 2001.

[14] M. R. Ganigi and B. A. Uralegaddi, "New criteria for meromorphic univalent functions," Bulletin Mathématique de la Société des Sciences Mathématiques de la République Socialiste de Roumanie. Nouvelle Série, vol. 33, no. 81, pp. 9-13, 1989.

[15] D. Yang, "On a class of meromorphic starlike multivalent functions," Bulletin of the Institute of Mathematics Academia Sinica, vol. 24, no. 2, pp. 151-157, 1996.

[16] A. Mohammed and M. Darus, "A new integral operator for meromorphic functions," Acta Universitatis Apulensis, no. 24, pp. 231-238, 2010.

[17] A. Mohammed and M. Darus, "Starlikeness properties of a new integral operator for meromorphic functions," Journal of Applied Mathematics, vol. 2011, Article ID 804150, 8 pages, 2011.

[18] A. Mohammed and M. Darus, "Some properties of certain integral operators on new subclasses of analytic functions with complex order," Journal of Applied Mathematics, vol. 2012, Article ID 161436, 9 pages, 2012.

[19] A. Mohammed and M. Darus, "The order of starlikeness of new p-valent meromorphic functions," International Journal of Mathematical Analysis, vol. 6, no. 27, pp. 1329-1340, 2012.

[20] A. Mohammed and M. Darus, "Integral operators on new families of meromorphic functions of complex order," Journal of Inequalities and Applications, vol. 2011, article 121, 12 pages, 2011. 


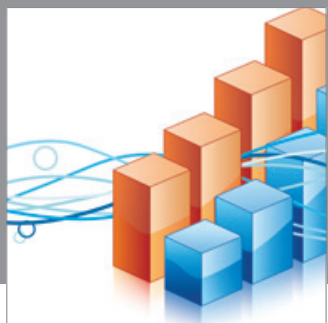

Advances in

Operations Research

mansans

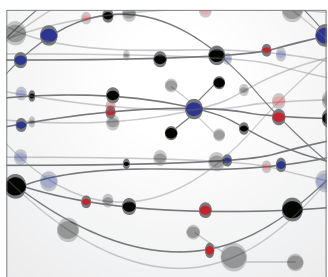

The Scientific World Journal
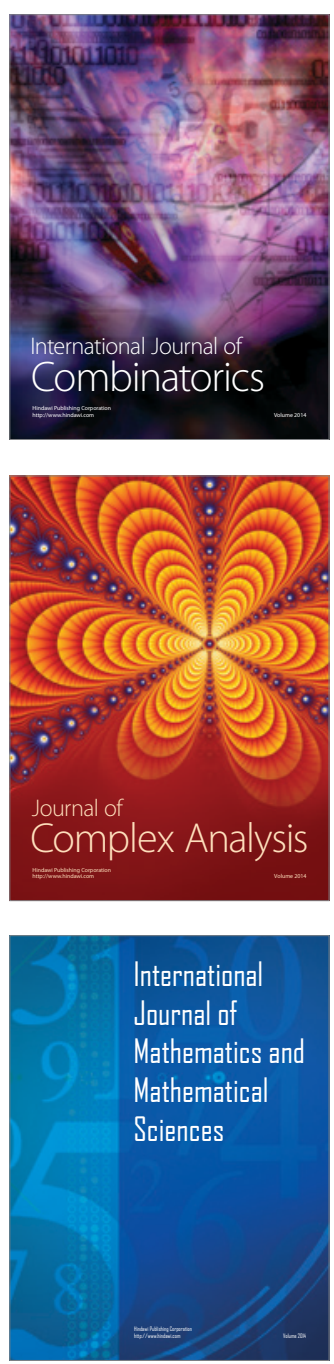
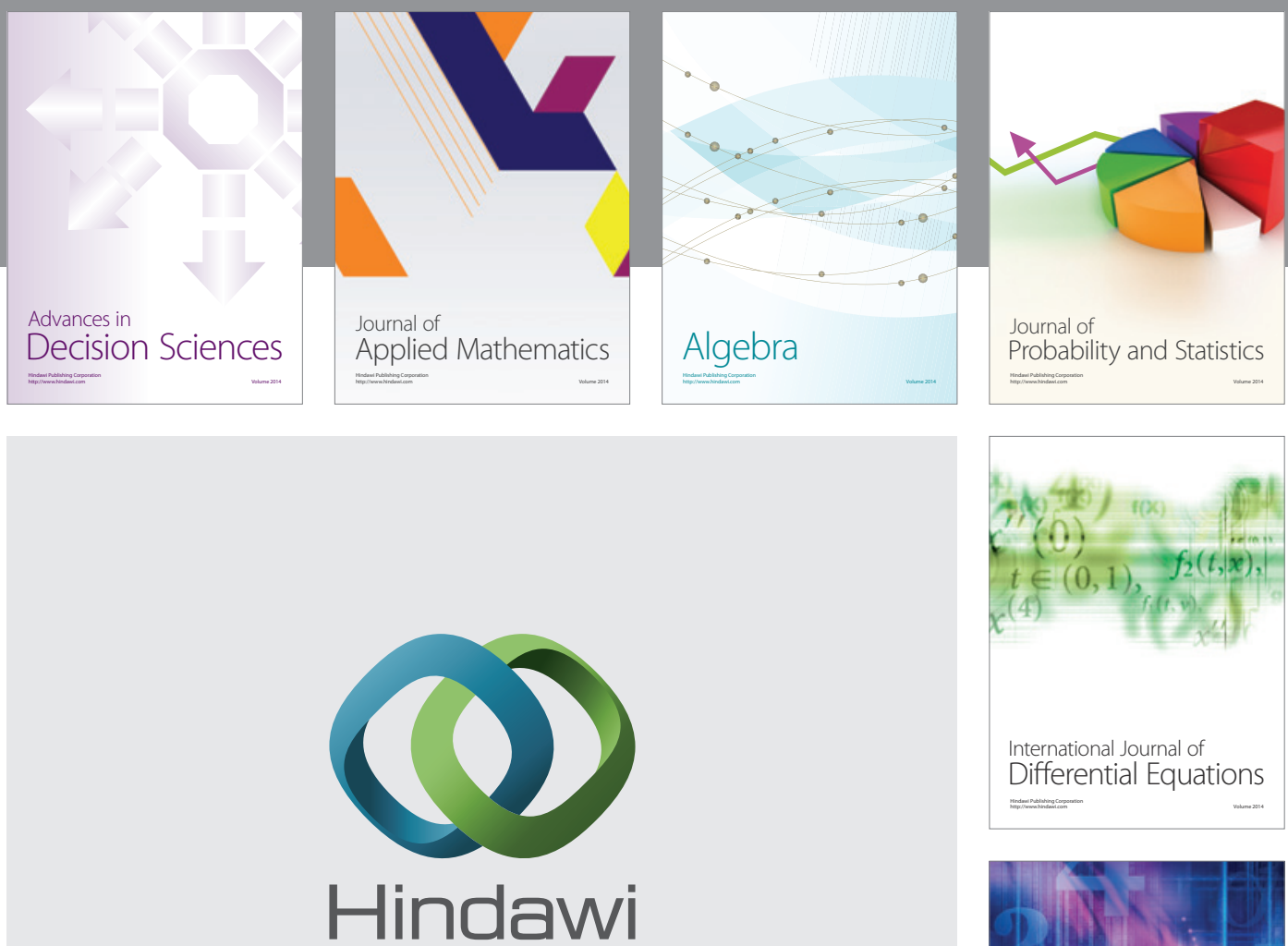

Submit your manuscripts at http://www.hindawi.com
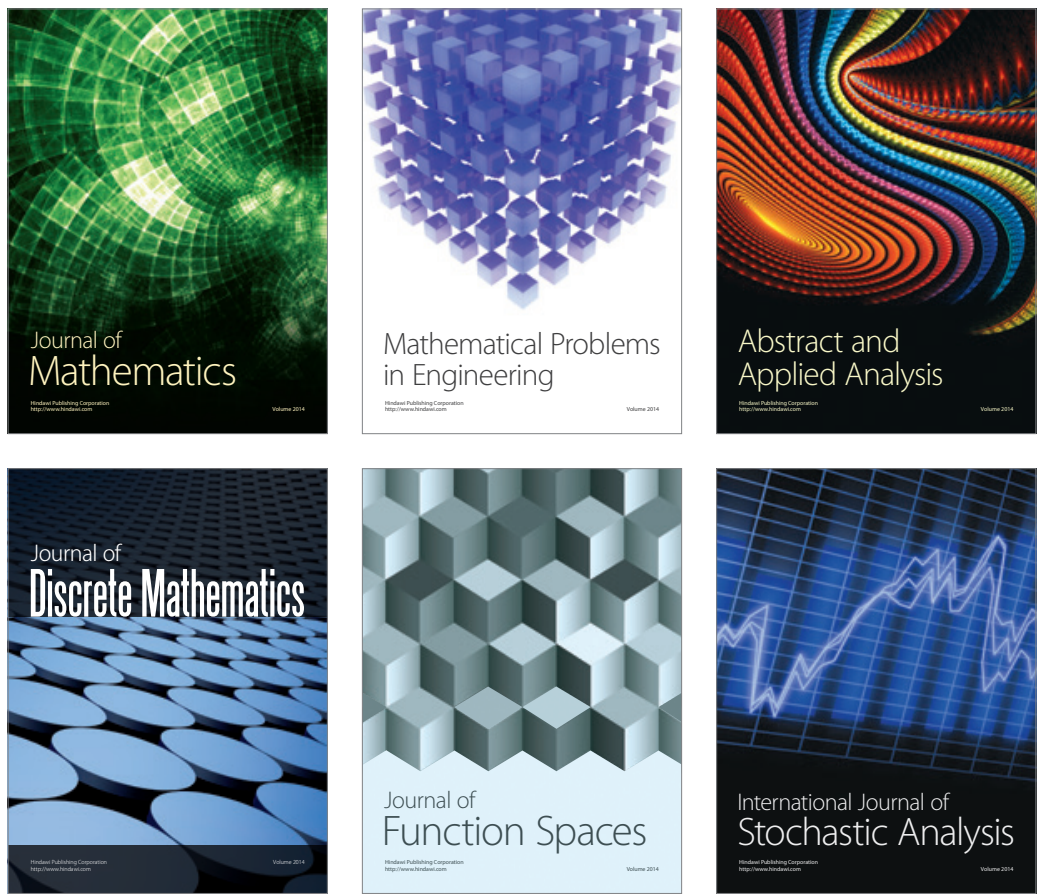

Journal of

Function Spaces

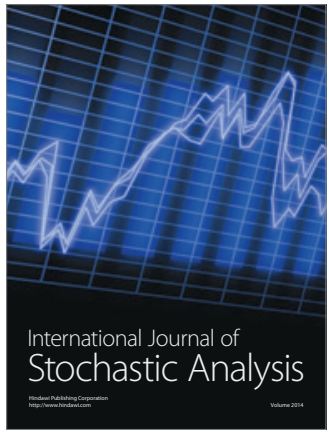

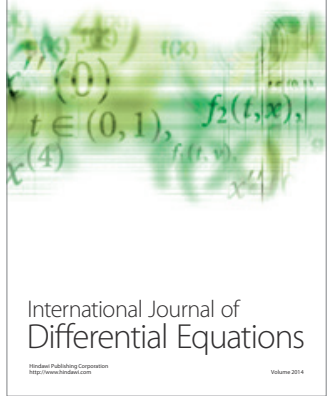
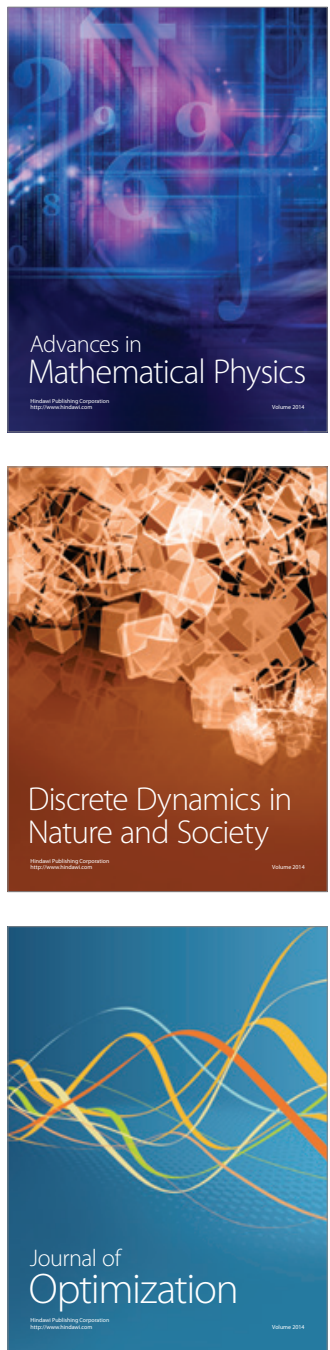